East European Journal of Physics

\title{
MODULATION INSTABILITY IN TWO COMPONENT BOSE-EINSTEIN CONDENSATE WITH DISSIPATION
}

\author{
Anatoly Ivashin*, Elena Marinenko \\ National Science Centre "Kharkov institute of physics and technology" \\ Akademichna str., 61108, Kharkiv, Ukraine \\ *E-mail: ivashin@kipt.kharkov.ua \\ Received November 13, 2018; revised February 4, 2019; accepted March 25, 2018
}

In this paper, we consider the effect of dissipation on the stability of relatively small perturbations of a binary mixture of a Bose Einstein condensate. Using the introduction of a dissipative function, the Gross-Pitaevskii equations for a two-component system are obtained taking into account dissipation. The influence of dissipative processes on the development of modulation instability in a spatially homogeneous two-component Bose-Einstein condensate is investigated. In contrast to the one-component Bose-Einstein condensate, where modulation instability occurs only with a negative interaction constant, in a two-component Bose-Einstein condensate the conditions for the occurrence of modulation instability significantly depend on the signs and magnitudes of the constant interactions between particles. The paper considers cases when the signs of constant interaction of particles of the same kind are the same and when they are opposite. In the first case, for the occurrence of instability, a certain condition must be satisfied for the magnitudes of the interaction constant, and in the second case, the instability region arises independently of the magnitudes of the interaction constant. In the approximation linear in the dissipation coefficient, the dependences of the values of the growth increment of oscillations and the wave number, at which the increment reaches the maximum value, are obtained, on the dissipative coefficient. It is shown that in this approximation the region of wave numbers at which instability occurs, and the value of the maximum growth increment does not change when taking into account dissipation. The effect of dissipation on these characteristics should be manifested when taking into account corrections that are quadratic in terms of the dissipation coefficient. In the region of wave numbers, where the system remains stable, the damping decrements of oscillations are calculated and it is shown that in this case, in the long-wavelength limit, the oscillation frequency linearly depends on the wave vector, as in the case of single-component condensate.

KEY WORDS: Bose Einstein two-component condensate, Gross-Pitaevskii equations, modulation instability

\section{МОДУЛЯЦІЙНА НЕСТІЙКІСТЬ У ДВОКОМПОНЕНТНОМУ БОЗЕ-ЕЙНШТЕЙНІВСЬКОМУ КОНДЕНСАТІ 3 ДИСИПАЦІЮ}

А.П. Івашин, О.Д. Маріненко

Національний науковий центр “Харківський фізико-технічний інститут”" 61108, м. Харків, вул. Академічна, 1

В даній роботі розглядається вплив дисипації на стійкість відносно малих збуджень бінарної суміші бозе-ейнштейнівського конденсату. За допомогою введення дисипативної функції отримані рівняння Гроса - Питаєвського для двокомпонентної системи з урахуванням дисипації. Досліджено вплив дисипативних процесів на розвиток модуляційної нестійкості у просторово-однорідному двокомпонентному бозе-ейнштейнівському конденсаті. На відміну від однокомпонентного бозеейнштейнівського конденсату, де модуляційна нестійкість виникає тільки при від`ємній константі взаємодії, в двокомпонентному бозе-ейнштейнівському конденсаті умови виникнення модуляційної нестійкості суттєво залежать від знаків і величин постійних взаємодії між частинками. В роботі розглянуті випадки, коли знаки постійних взаємодії частинок одного сорту однакові і коли протилежні. В першому випадку для виникнення нестійкості повинна виконуватись певна умова на співвідношення між величинами постійних взаємодії, а в другому випадку область нестійкості виникає незалежно від величини постійних взаємодії. В лінійному по коефіцієнту дисипації наближенні знайдені залежності величин інкремента наростання коливань і хвильового числа, при якому інкремент досягає максимального значення, від величини дисипативного коефіцієнта. Показано, що в цьому наближенні область хвильових чисел, при яких виникає нестійкість, $\mathrm{i}$ величина максимального інкремента наростання при урахуванні дисипації не змінюється. Вплив дисипації на ці характеристики повинен проявлятися при урахуванні квадратичних по коефіцієнту дисипації поправок. В області хвильових чисел, де система залишається стійкою, обчислені декременти затухання коливань і показано, що в цьому випадку, в довгохвильовому наближенні, частота коливань лінійно залежить від хвильового вектора, як і в разі однокомпонентного конденсату.

КЛЮЧОВІ СЛОВА: бозе - ейнштейнівській двокомпонентний конденсат, рівняння Гроса - Питаєвського, модуляційна нестійкість

\section{МОДУЛЯЦИОННАЯ НЕУСТОЙЧИВОСТЬ В ДВУХКОМПОНЕНТНОМ БОЗЕ-ЭЙНШТЕЙНОВСКОМ КОНДЕНСАТЕ С ДИССПАЦИЕЙ А.П. Ивашин, Е.Д. Мариненко}

Национальный научный иентр “Харьковский физико-технический институт” 61108, г. Харьков, ул. Академическая, 1

В настоящей работе рассматривается влияние диссипации на устойчивость относительно малых возмущений бинарной смеси бозе-эйнштейновского конденсата. С помощью введения диссипативной функции получены уравнения ГроссаПитаевского для двухкомпонентной системы с учётом диссипации. Исследовано влияние диссипативных процессов на 
развитие модуляционной неустойчивости в пространственно-однородном двухкомпонентном бозе-эйнштейновском конденсате. В отличие от однокомпонентного бозе-эйнштейновского конденсата, где модуляционная неустойчивость возникает только при отрицательной константе взаимодействия, в двухкомпонентном бозе-эйнштейновском конденсате условия возникновения модуляционной неустойчивости существенно зависят от знаков и величин постоянных взаимодействия между частицами. В работе рассмотрены случаи, когда знаки постоянных взаимодействия частиц одного сорта одинаковы и когда противоположны. В первом случае для возникновения неустойчивости должно выполняться определённое условие на величины постоянных взаимодействия, а во втором случае область неустойчивости возникает независимо от величин постоянных взаимодействия. В линейном по коэффициенту диссипации приближении получены зависимости величин инкремента нарастания колебаний и волнового числа, при котором инкремент достигает максимального значения, от величины диссипативного коэффициента. Показано, что в этом приближении область волновых чисел, при которых возникает неустойчивость, и величина максимального инкремента нарастания при учёте диссипации не меняется. Влияние диссипации на эти характеристики должно проявляться при учёте квадратичных по коэффициенту диссипации поправок. В области волновых чисел, где система остаётся устойчивой, вычислены декременты затухания колебаний и показано, что в этом случае, в длинноволновом пределе, частота колебаний линейно зависит от волнового вектора, как и в случае однокомпонентного конденсата.

КЛЮЧЕВЫЕ СЛОВА: бозе-эйнштейновский двухкомпонентный конденсат, уравнения Гросса-Питаевского, модуляционная неустойчивость

Атомарный бозе-эйнштейновский конденсат (БЭК) это перспективный объект для изучения модуляционной неустойчивости (МН), приводящей к формированию в однородной среде доменных структур, темных и светлых солитонов, квантовых вихрей при стремлении системы к равновесию [1-3]. Модуляционная неустойчивость это общее явление для нелинейных уравнений, в которых слабые пространственные возмущения в определенном диапазоне волн растут экспоненциально в цепочку локализованных волн в результате взаимодействия нелинейности и дисперсии [4,5].

В БЭК интерес к модуляционной неустойчивости был вызван экспериментальным наблюдением динамики коллапса и генерацией цепочки солитонов $[5,6]$. В однокомпонентном БЭК с отрицательной константой связи экспериментально наблюдались множественные чередующиеся долгоживущие домены [7]. Многие теоретические работы были посвящены изучению формирования структур, вызванных МН $[8,9]$.

Двухкомпонентные БЭК представляют собой более богатую систему для изучения МН, и существенно отличаются от однокомпонентного случая, где МН возникает только при отрицательной константе связи между частицами конденсата. Впервые МН в двухкомпонентных БЭК обсуждалась в работе [10]. Первые экспериментальные работы проводились в системах положительной константой межатомного взаимодействия. В теоретических работах [11-14], в рамках уравнения Гросса-Питаевского (ГП), изучены условия возникновения МН при различных наборах параметров, характеризующих двухкомпонентный конденсат. Были рассмотрены случаи, как с положительными, так и с отрицательными межатомными взаимодействиями внутри компонент и между компонентами.

Уравнение ГП, хорошо описывающее физические процессы в БЭК при нулевой температуре, так же хорошо описывает процессы в этих системах вплоть до $0.5 T_{c}$. Однако учет диссипации в таких системах, как правило, не производился, т.к. предполагалось, что при низких температурах динамика полностью определяется частицами, находящимися в конденсате. Но при отличной от нуля температуре уже присутствуют процессы с диссипацией энергии, обусловленные взаимодействием конденсатных частиц с частицами вне конденсата. К тому же, надконденсатные частицы образуются не только за счет температурных эффектов, но и из-за истощения конденсата, обусловленного межчастичным взаимодействием. Поскольку обычное уравнение ГП не описывает диссипативные процессы, необходимо либо модифицировать его, либо рассматривать совместно динамические уравнения для конденсата и надконденсатных квазичастиц. При выводе уравнений ГП мы будем следовать работе [15]. Целью данной статьи является учет вклада диссипации в нелинейную динамику конденсата, а именно, нахождение максимального инкремента колебаний МН и определение области волновых векторов, при которых МН существует. При получении уравнения ГП мы вводим в исходный лагранжиан диссипативную функцию. Далее анализируем динамику волновых процессов и условия возникновения МН с учетом диссипативных процессов. В статье система уравнений ГП для двухкомпонентного БЭК, представлена в гидродинамическом виде. В [15] это позволило лучше прояснить смысл введенного диссипативного коэффициента $\gamma$. Получен спектр возбуждений при наличии диссипации в системе и показано, как он зависит от знаков констант связи. В частности, при положительном значении постоянных взаимодействия в каждой компоненте, могут существовать как затухающие колебания, так и возникать модуляционная неустойчивость. Когда в одной из компонент постоянная взаимодействия отрицательна, а в другой - положительна, условия для развития МН, приведенные в [16], перестают быть необходимыми. В обоих случаях мы находим максимальный инкремент и определяем границы неустойчивости в пространстве волновых чисел.

В первом разделе работы получена система уравнений Гросса-Питаевского для двухкомпонентного БЭК с учетом диссипации. Во втором разделе уравнения ГП представлены в гидродинамической форме и, в предположении малости возбуждений и слабой диссипации, получено дисперсионное уравнение. В третьем 
разделе исследованы решения дисперсионного уравнения и определены декременты затухания, обусловленные наличием диссипации в системе. В четвертом разделе дан анализ полученного спектра при условии развития МН, рассмотрены случаи, когда положительны обе константы взаимодействия, в первой и второй компоненте, а также, когда во второй компоненте константа взаимодействия отрицательна. Найден максимальный инкремент колебаний, определены границы области МН и найдены соответствующие граничные волновые вектора. В заключении представлены выводы.

\section{ПОЛУЧЕНИЕ ДИНАМИЧЕСКИХ УРАВНЕНИЙ ДЛЯ ДВУХКОМПОНЕНТНОЙ СИСТЕМЫ С УЧЕТОМ ДИССИПАЦИИ}

Диссипативные процессы в двухкомпонентном БЭК, состоящем из частиц двух сортов атомов с массами $m_{1}, m_{2}$ и плотностями $n_{1}, n_{2}$ можно учесть феноменологически в рамках лагранжева формализма с помощью введения диссипативной функции [15]. В этом случае уравнение Эйлера-Лагранжа будет иметь вид

$$
\frac{d}{d t} \frac{\partial \Lambda}{\partial \dot{\psi}_{k}}-\frac{\partial \Lambda}{\partial \psi_{k}}+\nabla \frac{\partial \Lambda}{\partial \nabla \psi_{k}}=-\frac{\partial D}{\partial \dot{\psi}_{k}}
$$

здесь плотность функции Лагранжа $\Lambda$ имеет вид:

$$
\Lambda=\sum_{k}\left[i \frac{\hbar}{2}\left(\psi_{k}^{*} \dot{\psi}_{k}-\dot{\psi}_{k}^{*} \psi_{k}\right)-\frac{\hbar^{2}}{2 m_{k}}\left|\nabla \psi_{k}\right|^{2}-\left[V_{k}(\mathbf{r})-\mu_{k}\right]\left|\psi_{k}\right|^{2}\right]-\frac{1}{2} \sum_{i, k} g_{i k}\left|\psi_{i}\right|^{2}\left|\psi_{k}\right|^{2}
$$

где индексы $i, k=1,2$ используются здесь для обозначения компонент конденсата.

Квадратичная по скорости диссипативная функция $D$ может быть записана в виде [15]

$$
D=\hbar \sum_{i, k} \gamma_{i k} \dot{\psi}_{i}^{*} \dot{\psi}_{k}
$$

где $\gamma_{i k}$ - безразмерные диссипативные коэффициенты, причем $\gamma_{i k}=\gamma_{k i}^{*}$.

В комплексных функциях можно выделить модули $f_{i}$ и фазы $\varphi_{i}$, записав их в виде $\psi_{i}(\mathbf{r}, t)=f_{i}(\mathbf{r}, t) \exp \left[i \varphi_{i}(\mathbf{r}, t)\right]$. Тогда плотности числа частиц $n_{i}(\mathbf{r}, t)$ и скорости $\mathbf{v}_{i}(\mathbf{r}, t)$ каждой компоненты выражаются через модуль и фазу

$$
n_{i}(\mathbf{r}, t)=f_{i}^{2}(\mathbf{r}, t), \quad \mathbf{v}_{i}(\mathbf{r}, t)=\frac{\hbar}{m_{i}} \nabla \varphi_{i}(\mathbf{r}, t) .
$$

В пространственно-однородном состоянии химические потенциалы $\mu_{1}, \mu_{2}$ связаны с равновесными плотностями [16]

$$
\mu_{1}=g_{1} n_{10}+g_{12} n_{20}, \quad \mu_{2}=g_{2} n_{20}+g_{21} n_{10} .
$$

Уравнение (1) для Бозе-Эйнштейновского конденсата приводит к уравнения Гросса - Питаевского для макроскопических волновых функций $\psi_{1}(\mathbf{r}, t), \psi_{2}(\mathbf{r}, t)[16]$

$$
\begin{aligned}
i \hbar \dot{\psi}_{1}(\mathbf{r}, t)-\hbar \gamma_{1} \dot{\psi}_{1}(\mathbf{r}, t) & =-\frac{\hbar^{2}}{2 m_{1}} \Delta \psi_{1}(\mathbf{r}, t)+g_{1} \psi_{1}(\mathbf{r}, t)\left|\psi_{1}(\mathbf{r}, t)\right|^{2}+g_{12} \psi_{1}(\mathbf{r}, t)\left|\psi_{2}(\mathbf{r}, t)\right|^{2}-\mu_{1} \psi_{1}(\mathbf{r}, t), \\
i \hbar \dot{\psi}_{2}(\mathbf{r}, t)-\hbar \gamma_{2} \dot{\psi}_{2}(\mathbf{r}, t) & =-\frac{\hbar^{2}}{2 m_{2}} \Delta \psi_{2}(\mathbf{r}, t)+g_{2} \psi_{2}(\mathbf{r}, t)\left|\psi_{2}(\mathbf{r}, t)\right|^{2}+g_{21} \psi_{2}(\mathbf{r}, t)\left|\psi_{1}(\mathbf{r}, t)\right|^{2}-\mu_{2} \psi_{2}(\mathbf{r}, t) .
\end{aligned}
$$

В уравнениях (6) взаимодействие между компонентами конденсата осуществляется посредством их плотностей $\left|\psi_{k}(\mathbf{r}, t)\right|^{2}$. Мы положили $\gamma_{12}=0$, исключив, таким образом, взаимное влияние компонент конденсата через диссипацию и существенно упростив в дальнейшем решение полученной системы уравнений. 
Выделим в уравнениях (6) реальную и мнимую части, совершив в них предварительно переход от функций $\psi_{i}$ к функциям $f_{i}$ и $\varphi_{i}$. Тогда система уравнений (6) примет вид

$$
\begin{aligned}
& f_{1} \dot{\varphi}_{1}+\gamma_{1} \dot{f}_{1}=\left(\hbar / 2 m_{1}\right)\left(\Delta f_{1}-f_{1}\left(\nabla \varphi_{1}\right)^{2}\right)-(1 / \hbar)\left(g_{1} f_{1} f_{1}^{2}+g_{12} f_{1} f_{2}^{2}\right)+(1 / \hbar) f_{1} \mu_{1} \\
& f_{2} \dot{\varphi}_{2}+\gamma_{2} \dot{f}_{2}=\left(\hbar / 2 m_{2}\right)\left(\Delta f_{2}-f_{2}\left(\nabla \varphi_{2}\right)^{2}\right)-(1 / \hbar)\left(g_{2} f_{2} f_{2}^{2}+g_{21} f_{2} f_{1}^{2}\right)+(1 / \hbar) f_{2} \mu_{2} \\
& \hbar \dot{f}_{1}-\hbar \gamma_{1} f_{1} \dot{\varphi}_{1}=-\left(\hbar^{2} / 2 m_{1}\right)\left(2 \nabla f_{1} \nabla \varphi_{1}+f_{1} \Delta \varphi_{1}\right) \\
& \hbar \dot{f}_{2}-\hbar \gamma_{2} f_{2} \dot{\varphi}_{2}=-\left(\hbar^{2} / 2 m_{2}\right)\left(2 \nabla f_{2} \nabla \varphi_{2}+f_{2} \Delta \varphi_{2}\right)
\end{aligned}
$$

Мы получили систему уравнений для четырех неизвестных величин $f_{1}, f_{2}, \varphi_{1}, \varphi_{2}$, которая зависит от девяти параметров: от масс двух компонент конденсата $m_{1}$ и $m_{2}$, трех констант связи $g_{1}, g_{2}, g_{12}$, двух химических потенциалов $\mu_{1}$ и $\mu_{2}$, и двух коэффициентов диссипации $\gamma_{1}$ и $\gamma_{2}$. В однокомпонентном конденсате число параметров равно четырем. Таким образом, нелинейная динамика в двухкомпонентном случае определяется большим числом параметров. Это приводит к большему разнообразию физических явлений в двухкомпонентных системах с БЭК по сравнению с однокомпонентными системами.

\section{ГИДРОДИНАМИЧЕСКАЯ ФОРМА УРАВНЕНИЙ ГРОССА-ПИТАЕВСКОГО И ПОЛУЧЕНИЕ} ДИСПЕРСИОННОГО УРАВНЕНИЯ

В соответствии с (4) систему уравнений (7) можно переписать в виде

$$
\begin{aligned}
& \nabla \dot{n}_{1}+\nabla\left(\nabla \cdot\left(n_{1} \mathbf{v}_{1}\right)\right)=2 \gamma_{1} n_{1} m_{1} \dot{\mathbf{v}}_{1} / \hbar \\
& \nabla \dot{n}_{2}+\nabla\left(\nabla \cdot\left(n_{2} \mathbf{v}_{2}\right)\right)=2 \gamma_{2} n_{2} m_{2} \dot{\mathbf{v}}_{2} / \hbar \\
& m_{1} \dot{\mathbf{v}}_{1}+\left(\hbar \gamma_{1} /\left(2 n_{1}\right)\right) \nabla \dot{n}_{1}=-\nabla \tilde{\mu}_{1} \\
& m_{2} \dot{\mathbf{v}}_{2}+\left(\hbar \gamma_{2} /\left(2 n_{2}\right)\right) \nabla \dot{n}_{2}=-\nabla \tilde{\mu}_{2}
\end{aligned}
$$

где $\tilde{\mu}_{1}=g_{1} n_{1}+g_{12} n_{2}-\left(\hbar^{2} / 2 m_{1}\right) \Delta \sqrt{n_{1}} / \sqrt{n_{1}}$ и $\tilde{\mu}_{2}=g_{2} n_{2}+g_{21} n_{1}-\left(\hbar^{2} / 2 m_{2}\right) \Delta \sqrt{n_{2}} / \sqrt{n_{2}}$ - химические потенциалы для первой и второй компоненты конденсата соответственно с учетом квантового давления. При переходе к гидродинамическому виду уравнений мы пренебрегли квадратичными членами, пропорциональными $\left(\nabla \varphi_{i}\right)^{2}$.

В полученной системе гидродинамических уравнений для двухкомпонентного БЭК (8), первые два уравнения представляют собой модифицированные уравнения непрерывности для каждой компоненты конденсата соответственно, а два оставшихся, это уравнения Эйлера с учетом диссипации.

Положим в (8) $n_{i}=n_{i 0}+\delta n_{i}$ и $\mathbf{v}_{i}=\mathbf{v}_{i 0}+\delta \mathbf{v}_{i}$, где плотности $n_{i 0}$ и скорости $\mathbf{v}_{i 0}$ соответствуют основному состоянию конденсата, а $\delta n_{i}$ и $\delta \mathbf{v}_{i}$ - малые возмущения основного состояния конденсата, зависящие от времени и пространственной координаты $\delta n_{i}=\delta n_{i}(\mathbf{r}, t), \delta \mathbf{v}_{i}=\delta \mathbf{v}_{i}(\mathbf{r}, t)$. Считая, что исходно конденсаты покоятся, величину $\mathbf{v}_{i 0}$ полагаем равной нулю, а величину $\delta \mathbf{v}_{i}$ будем писать в виде $\mathbf{v}_{i}$, помня, что она одного порядка малости с $\delta n_{i}$. Линеаризуя систему (8) получим

$$
\begin{aligned}
& \nabla \delta \dot{n}_{1}+\nabla\left(\nabla \cdot\left(n_{10} \mathbf{v}_{1}\right)\right)=2 \gamma_{1} n_{10} m_{1} \dot{\mathbf{v}}_{1} / \hbar \\
& \nabla \delta \dot{n}_{2}+\nabla\left(\nabla \cdot\left(n_{20} \mathbf{v}_{2}\right)\right)=2 \gamma_{2} n_{20} m_{2} \dot{\mathbf{v}}_{2} / \hbar \\
& m_{1} \dot{\mathbf{v}}_{1}+\left(\hbar \gamma_{1} /\left(2 n_{10}\right)\right) \nabla \delta \dot{n}_{1}=-\nabla \delta \tilde{\mu}_{1} \\
& m_{2} \dot{\mathbf{v}}_{2}+\left(\hbar \gamma_{2} /\left(2 n_{20}\right)\right) \nabla \delta \dot{n}_{2}=-\nabla \delta \tilde{\mu}_{2}
\end{aligned}
$$

где $\delta \tilde{\mu}_{1}=g_{1} \delta n_{1}+g_{12} \delta n_{2}-\left(\hbar^{2} /\left(4 m_{1} n_{10}\right)\right) \Delta \delta n_{1}$ и $\delta \tilde{\mu}_{2}=g_{2} \delta n_{2}+g_{12} \delta n_{1}-\left(\hbar^{2} /\left(4 m_{2} n_{20}\right)\right) \Delta \delta n_{2}$.

Будем искать решение системы уравнений (9) в виде

$$
\delta n, \mathbf{v} \sim e^{-i(\omega t-\mathbf{k} \cdot \mathbf{r})} .
$$

В этом случае система уравнений относительно $\delta n_{1}, \delta n_{1}, \mathbf{v}_{1}, \mathbf{v}_{2}$ приобретает вид 


$$
\begin{aligned}
& \omega \mathbf{k} \delta n_{1}+\left(2 n_{10} m_{1} / \hbar^{2}\right)\left(i \hbar \omega \gamma_{1}-\varepsilon_{1}^{0}\right) \mathbf{v}_{1}=0 \\
& \omega \mathbf{k} \delta n_{2}+\left(2 n_{20} m_{2} / \hbar^{2}\right)\left(i \hbar \omega \gamma_{2}-\varepsilon_{2}^{0}\right) \mathbf{v}_{2}=0 \\
& \left(i \mathbf{k} / 2 n_{10}\right)\left(2 g_{1} n_{10}+\varepsilon_{1}^{0}-i \hbar \omega \gamma_{1}\right) \delta n_{1}+i \mathbf{k} g_{12} \delta n_{2}-i \omega m_{1} \mathbf{v}_{1}=0 \\
& i \mathbf{k} g_{12} \delta n_{1}+\left(i \mathbf{k} / 2 n_{20}\right)\left(2 g_{2} n_{20}+\varepsilon_{2}^{0}-i \hbar \omega \gamma_{2}\right) \delta n_{2}-i \omega m_{2} \mathbf{v}_{2}=0
\end{aligned},
$$

где $\varepsilon_{i}^{0}=(\hbar k)^{2} /\left(2 m_{i}\right)$.

Приравнивая детерминант системы (11) нулю получим дисперсионное уравнение

$$
\begin{aligned}
& (\hbar \omega)^{4}+2 i(\hbar \omega)^{3}\left\{\gamma_{1}\left(n_{10} g_{1}+\varepsilon_{1}^{0}\right)+\gamma_{2}\left(n_{20} g_{2}+\varepsilon_{2}^{0}\right)\right\}-(\hbar \omega)^{2}\left\{\varepsilon_{1}^{2}+\varepsilon_{2}^{2}\right\}+ \\
& +2 i(\hbar \omega)\left\{2 n_{10} n_{20} g_{12}^{2}\left(\varepsilon_{1}^{0} \gamma_{2}+\varepsilon_{2}^{0} \gamma_{1}\right)-\gamma_{1} \varepsilon_{2}^{2}\left(g_{1} n_{10}+\varepsilon_{1}^{0}\right)-\gamma_{2} \varepsilon_{1}^{2}\left(g_{2} n_{20}+\varepsilon_{2}^{0}\right)\right\}+\left\{\varepsilon_{1}^{2} \varepsilon_{2}^{2}-4 n_{10} n_{20} g_{12}^{2} \varepsilon_{1}^{0} \varepsilon_{2}^{0}\right\}=0
\end{aligned}
$$

Здесь введено обозначение $\varepsilon_{i}^{2}=\left(2 g_{i} n_{i 0}+\varepsilon_{i}^{0}\right) \varepsilon_{i}^{0}$. Мы получили уравнение четвертого порядка относительно $\hbar \omega$ с комплексными коэффициентами при линейном и кубическом члене. Решение этого уравнения будем искать, используя малость диссипативных коэффициентов $\gamma_{1}, \gamma_{2}$.

Перепишем уравнение (12) в виде

\section{РЕШЕНИЕ ДИСПЕРСИОННОГО УРАВНЕНИЯ}

$$
x^{4}+a x^{3}+b x^{2}+c x+d=0
$$

где $x \equiv \hbar \omega$, а коэффициенты $a, b, c, d$ имеют вид

$$
\begin{aligned}
& a=2 i\left\{\gamma_{1}\left(n_{10} g_{1}+\varepsilon_{1}^{0}\right)+\gamma_{2}\left(n_{20} g_{2}+\varepsilon_{2}^{0}\right)\right\} \\
& b=-\left(\varepsilon_{1}^{2}+\varepsilon_{2}^{2}\right) \\
& c=2 i\left\{2 n_{10} n_{20} g_{12}^{2}\left(\varepsilon_{1}^{0} \gamma_{2}+\varepsilon_{2}^{0} \gamma_{1}\right)-\gamma_{1} \varepsilon_{2}^{2}\left(g_{1} n_{10}+\varepsilon_{1}^{0}\right)-\gamma_{2} \varepsilon_{1}^{2}\left(g_{2} n_{20}+\varepsilon_{2}^{0}\right)\right\} \\
& d=\varepsilon_{1}^{2} \varepsilon_{2}^{2}-4 n_{10} n_{20} g_{12}^{2} \varepsilon_{1}^{0} \varepsilon_{2}^{0}
\end{aligned}
$$

Из вида коэффициентов (14) следует, что знак межкомпонентного взаимодействия не влияет спектр колебаний в двухкомпонентном БЭК. Будем искать решение уравнения (13) в линейном по коэффициенту диссипации $\gamma$ приближении (как показано в [15], для атомарных конденсатов с плотностью числа частиц $n_{0} \approx 10^{15} \mathrm{~cm}^{-3}$ диссипативный коэффициент $\gamma \approx 10^{-4}$ ). Поскольку коэффициенты $а$ и $c$ линейны по $\gamma_{1}$ и $\gamma_{2}$, ищем решение в виде

$$
x=x_{0}+\gamma_{1} A+\gamma_{2} B
$$

где $x_{0}$ - решение дисперсионного уравнения (11) в отсутствие диссипации

$$
x_{0}^{4}+b x_{0}^{2}+d=0
$$

а $A$ и $B$ искомые константы, $\left(x_{0} \equiv \hbar \omega_{0}\right)$.

Решения в отсутствие диссипации известны [16] и имеют вид

$$
\left(\hbar \omega_{0}^{ \pm}\right)^{2}=\frac{\varepsilon_{1}^{2}+\varepsilon_{2}^{2}}{2} \pm \sqrt{\left(\frac{\varepsilon_{1}^{2}-\varepsilon_{2}^{2}}{2}\right)^{2}+4 n_{10} n_{20} g_{12}^{2} \varepsilon_{1}^{0} \varepsilon_{2}^{0}} .
$$

В отсутствие диссипации в двухкомпонентном конденсате имеются две ветви колебаний $\omega_{0}^{+}$и $\omega_{0}^{-}$, которые соответствуют разным знакам перед корнем. При отсутствии взаимодействия между конденсатами $\left(g_{12}=0\right)$, из (17) следует 


$$
\left(\hbar \omega_{0}^{+}\right)^{2}=\varepsilon_{1}^{2}=\left(2 g_{1} n_{10}+\varepsilon_{1}^{0}\right) \varepsilon_{1}^{0} \text { и }\left(\hbar \omega_{0}^{-}\right)^{2}=\varepsilon_{2}^{2}=\left(2 g_{2} n_{20}+\varepsilon_{2}^{0}\right) \varepsilon_{2}^{0} .
$$

Это хорошо известный результат, полученный Боголюбовым для однокомпонентного конденсата [16]. В случае действительных частот, $\left(\hbar \omega_{0}^{ \pm}\right)^{2}>0$, при соотношении параметров $g_{1} g_{2}>\left(g_{12}\right)^{2}$ в (17), система будет устойчива, и в ней будут существовать две ветви колебаний с частотами $\omega_{0}^{ \pm}$. Анализ устойчивости системы относительно малых возмущений в случае, когда соотношение между параметрами $g_{1} g_{2}<\left(g_{12}\right)^{2}$, приводит, согласно (17), к наличию модуляционной неустойчивости, $\left(\hbar \omega_{0}^{ \pm}\right)^{2}<0[17]$.

Учтем наличие диссипации в системе. Подставляя выражение $x=x_{0}+\gamma_{1} A+\gamma_{2} B$ в уравнение (13) и приравнивая нулю коэффициенты при $\gamma_{1}$ и $\gamma_{2}$ найдем коэффициенты $A$ и $B$

$$
\begin{aligned}
& A=-\frac{i}{2} \frac{\left(n_{10} g_{1}+\varepsilon_{1}^{0}\right)\left(\hbar \omega_{0}\right)^{2}+2 n_{10} n_{20} g_{12}^{2} \varepsilon_{2}^{0}-\varepsilon_{2}^{2}\left(n_{10} g_{1}+\varepsilon_{1}^{0}\right)}{2\left(\hbar \omega_{0}\right)^{2}-\left(\varepsilon_{1}^{2}+\varepsilon_{2}^{2}\right)} \\
& B=-\frac{i}{2} \frac{\left(n_{20} g_{2}+\varepsilon_{2}^{0}\right)\left(\hbar \omega_{0}\right)^{2}+2 n_{10} n_{20} g_{12}^{2} \varepsilon_{1}^{0}-\varepsilon_{1}^{2}\left(n_{20} g_{2}+\varepsilon_{2}^{0}\right)}{2\left(\hbar \omega_{0}\right)^{2}-\left(\varepsilon_{1}^{2}+\varepsilon_{2}^{2}\right)}
\end{aligned} .
$$

В результате получим решение уравнения (13) в линейном по параметрам $\gamma_{1}, \gamma_{2}$ приближении

$$
\begin{aligned}
& \left(\hbar \omega^{ \pm}\right)=\left(\hbar \omega_{0}^{ \pm}\right)-\frac{i \gamma_{1}}{2} \frac{\left(n_{10} g_{1}+\varepsilon_{1}^{0}\right)\left(\hbar \omega_{0}^{ \pm}\right)_{ \pm}{ }^{2}+2 n_{10} n_{20} g_{12}^{2} \varepsilon_{2}^{0}-\varepsilon_{2}^{2}\left(n_{10} g_{1}+\varepsilon_{1}^{0}\right)}{2\left(\hbar \omega_{0}^{ \pm}\right)^{2}-\left(\varepsilon_{1}^{2}+\varepsilon_{2}^{2}\right)}- \\
& -\frac{i \gamma_{2}}{2} \frac{\left(n_{20} g_{2}+\varepsilon_{2}^{0}\right)\left(\hbar \omega_{0}\right)_{ \pm}{ }^{2}+2 n_{10} n_{20} g_{12}^{2} \varepsilon_{1}^{0}-\varepsilon_{1}^{2}\left(n_{20} g_{2}+\varepsilon_{2}^{0}\right)}{2\left(\hbar \omega_{0}^{ \pm}\right)^{2}-\left(\varepsilon_{1}^{2}+\varepsilon_{2}^{2}\right)}
\end{aligned} .
$$

где $\hbar \omega_{0}^{+}$и $\hbar \omega_{0}^{-}$соответствует 1-ой, и 2-ой ветви колебаний из (17).

В случае действительных частот, т.е. при $\left(\hbar \omega_{0}^{ \pm}\right)^{2}>0$, система устойчива, и в ней существуют две ветви затухающих во времени колебаний с частотами $\omega^{ \pm}=\omega_{0}^{ \pm}-i \Gamma^{ \pm}$, где $\Gamma^{ \pm}=\Gamma_{1}^{ \pm}+\Gamma_{2}^{ \pm}$

$$
\begin{aligned}
& \Gamma_{1}^{ \pm}=\frac{\gamma_{1}}{2 \hbar} \frac{\left(n_{10} g_{1}+\varepsilon_{1}^{0}\right)\left(\hbar \omega_{0}^{ \pm}\right)^{2}+2 n_{10} n_{20} g_{12}^{2} \varepsilon_{2}^{0}-\varepsilon_{2}^{2}\left(n_{10} g_{1}+\varepsilon_{1}^{0}\right)}{2\left(\hbar \omega_{0}^{ \pm}\right)^{2}-\left(\varepsilon_{1}^{2}+\varepsilon_{2}^{2}\right)} \\
& \Gamma_{2}^{ \pm}=\frac{\gamma_{2}}{2 \hbar} \frac{\left(n_{20} g_{2}+\varepsilon_{2}^{0}\right)\left(\hbar \omega_{0}^{ \pm}\right)^{2}+2 n_{10} n_{20} g_{12}^{2} \varepsilon_{1}^{0}-\varepsilon_{1}^{2}\left(n_{20} g_{2}+\varepsilon_{2}^{0}\right)}{2\left(\hbar \omega_{0}^{ \pm}\right)^{2}-\left(\varepsilon_{1}^{2}+\varepsilon_{2}^{2}\right)}
\end{aligned} .
$$

Решения уравнения (13) будут иметь вид $\delta n, \mathbf{v} \sim e^{-\Gamma t} e^{-i\left(\omega_{0} t-\mathbf{k} \cdot \mathbf{r}\right)}$.

\section{ВОЗНИКНОВЕНИЕ МН В БЭК ПРИ РАЗНЫХ ЗНАКАХ \\ ПОСТОЯННЫХ ВЗАИМОДЕЙСТВИЯ КОМПОНЕНТ}

В дальнейшем, положим для простоты анализа $m_{1}=m_{2}=m, \gamma_{1}=\gamma_{2}=\gamma$. Тогда выражения (17), (20) существенно упрощаются и принимают компактный вид

$$
\hbar \omega^{ \pm}=\hbar \omega_{0}^{ \pm}-i \hbar \Gamma^{ \pm}, \Gamma^{ \pm}=\frac{\gamma}{2 \hbar}\left(\varepsilon_{0}+\frac{\left(\hbar \omega_{0}^{ \pm}\right)^{2}}{\varepsilon_{0}}\right),
$$

где

$$
\left(\hbar \omega_{0}^{ \pm}\right)^{2}=\mathcal{E}_{0}\left(\varepsilon_{0}+n_{10} g_{1}+n_{20} g_{2} \pm \sqrt{\left(n_{10} g_{1}+n_{20} g_{2}\right)^{2}+4 n_{10} n_{20}\left(g_{12}^{2}-g_{1} g_{2}\right)}\right) .
$$

Запишем эти выражения в безразмерном виде. Для этого в (22) введем обозначения $E^{ \pm} \equiv\left(\hbar \omega^{ \pm}\right) /\left(n_{10} g_{1}\right)$, $E_{0}^{ \pm} \equiv\left(\hbar \omega_{0}^{ \pm}\right) /\left(n_{10} g_{1}\right), \chi \equiv\left(n_{20} g_{2}\right) /\left(n_{10} g_{1}\right)$ и $\tilde{k}^{2} \equiv \hbar^{2} k^{2} /\left(2 m n_{10} g_{1}\right)$. В итоге получим выражение для зависимости безразмерной энергии колебаний $E$ от безразмерного волнового вектора $\tilde{k}$

$$
E^{ \pm}=E_{0}^{ \pm}-i \hbar \tilde{\Gamma}^{ \pm}, \tilde{\Gamma}^{ \pm}=\frac{\gamma}{2 \hbar}\left(\tilde{k}^{2}+\frac{\left(E_{0}^{ \pm}\right)^{2}}{\tilde{k}^{2}}\right)
$$


где

$$
\left(E_{0}^{ \pm}\right)^{2}=\tilde{k}^{2}\left(\tilde{k}^{2}+(1+\chi) \pm \sqrt{(1+\chi)^{2}+4 \chi \eta_{1}}\right) .
$$

Мы также ввели обозначение $\eta_{1} \equiv g_{12}^{2} /\left(g_{1} g_{2}\right)-1$.

Исследуем вопрос о модуляционной неустойчивости в системе. В пренебрежении диссипацией модуляционная неустойчивость возникает, когда выражение $(25)$ для $\left(E_{0}^{ \pm}\right)^{2}$ отрицательно

$$
\left(\tilde{k}^{2}+(1+\chi) \pm \sqrt{(1+\chi)^{2}+4 \chi \eta_{1}}\right)<0
$$

Для случая, когда постоянные взаимодействия между частицами в компонентах конденсата положительны, $g_{1}>0, g_{2}>0$, это возможно только для $\left(E_{0}^{-}\right)^{2}$ (второй ветви колебаний - $\omega_{0}^{-}$), так как выражение для $\left(E_{0}^{+}\right)^{2}$ ( первая ветвь колебаний - $\omega_{0}^{+}$) всегда положительно (МН отсутствует). Вторая ветвь $\omega_{0}^{-}$ становится отрицательной при $\eta_{1}>0\left(g_{12}^{2}-g_{1} g_{2}>0\right)$, для волновых векторов $\tilde{k}^{2}<\tilde{k}_{B 1}^{2}[12]$

$$
\tilde{k}_{B 1}^{2}=\sqrt{(1+\chi)^{2}+4 \chi \eta_{1}}-(1+\chi) \text {. }
$$

Здесь $\tilde{k}_{B 1}$ - это граничное значение волнового вектора, когда в системе присутствует МН.

Модуляционная неустойчивость при наличии диссипации определяется неравенством $\left(E^{-}\right)^{2}<0$ и, соответственно, граничные значения волновых векторов находятся из уравнения

$$
E^{-}=E_{0}^{-}-\frac{i \gamma}{2}\left(\tilde{k}^{2}+\frac{\left(E_{0}^{-}\right)^{2}}{\tilde{k}^{2}}\right)=0,
$$

где значения $E_{0}^{-}$берутся из уравнения (25). В итоге получаем, что в линейном приближении по параметру $\gamma$, граничное значение $\tilde{k}_{B 1}$ для существования МН остается таким же, как и в отсутствие диссипации (27). Запишем его, возвращаясь к размерным величинам [12]

$$
k_{B 1}^{2}=\left(2 m / \hbar^{2}\right)\left[\sqrt{\left(n_{10} g_{1}+n_{20} g_{2}\right)^{2}+4 n_{10} n_{20}\left(g_{12}^{2}-g_{1} g_{2}\right)}-\left(n_{10} g_{1}+n_{20} g_{2}\right)\right] \text {. }
$$

Отрицательное значение $\left(E_{0}^{-}\right)^{2}$ (или $\left(\hbar \omega_{0}^{-}\right)^{2}$ ) говорит о том, что частота $\omega_{0}^{-}$- чисто мнимая величина, и ее можно представить как $\omega_{0}^{-}=i G_{0}$, где $G_{0}$, абсолютная величина $\omega_{0}^{-}$, представляет собой инкремент колебаний. Введем обозначения для инкрементов в отсутствие диссипации $\omega_{0}^{-}=i G_{01}$, и при наличии диссипации $\omega^{-}=i G_{1}$, в случае, когда константы взаимодействия положительны. Соответствующие им безразмерные величины будут $\tilde{G}_{01} \equiv \hbar G_{01} /\left(n_{10} g_{1}\right)$ и $\tilde{G}_{1} \equiv \hbar G_{1} /\left(n_{10} g_{1}\right)$. В случае, когда константы взаимодействия в компонентах конденсата имеют разные знаки будем использовать обозначения $G_{02}, \tilde{G}_{02}, \tilde{G}_{2}$ соответственно (см. далее). Из (25) следует, что в отсутствие диссипации

$$
\tilde{G}_{01}=\sqrt{\tilde{k}^{2}\left(\sqrt{(1+\chi)^{2}+4 \chi \eta_{1}}-\tilde{k}^{2}-(1+\chi)\right)} .
$$

И для величины $\tilde{G}_{1}$, согласно (28)

$$
\tilde{G}_{1}=\tilde{G}_{01}-\frac{\gamma}{2}\left(\tilde{k}^{2}+\frac{\left(E_{0}^{-}\right)^{2}}{\tilde{k}^{2}}\right)=0
$$

Выражение (31) определяет, в линейном приближении по диссипативному коэффициенту $\gamma$, зависимость инкремента колебаний от волнового вектора в диапазоне $0<\tilde{k}^{2}<\tilde{k}_{B 1}^{2}$. Таким образом, влияние диссипации на МН в рассматриваемом случае учитывается вторым слагаемым в $(31),(\gamma / 2)\left(2 \tilde{k}^{2}+(1+\chi)-\sqrt{(1+\chi)^{2}+4 \chi \eta_{1}}\right)$, в котором параметры $\chi$ и $\eta_{1}$ играют определяющую роль. Возвращаясь к размерным величинам получим

$$
G_{1}=G_{01}-\frac{\gamma}{2 \hbar}\left(2 \varepsilon_{0}+\left(n_{10} g_{1}+n_{2} g_{2}\right)-\sqrt{\left(n_{10} g_{1}+n_{2} g_{2}\right)^{2}+4 n_{10} n_{20} g_{1} g_{2} \eta_{1}}\right)
$$


где $G_{01}$ следует из (30)

$$
G_{01}=(1 / \hbar) \sqrt{\varepsilon_{0}\left(\sqrt{\left(n_{10} g_{1}+n_{20} g_{2}\right)^{2}+4 n_{10} n_{20} g_{1} g_{2} \eta_{1}}-\left(n_{10} g_{1}+n_{20} g_{2}\right)-\varepsilon_{0}\right)} .
$$

Определим моду колебаний, при которой инкремент МН будет максимальным. Она определяется условием

$$
\partial\left(E^{-}\right)^{2} / \partial \tilde{k}^{2}=0
$$

где $E^{-}$определяется формулой (28). Соответствующее значение волнового вектора равно

$$
\left.\tilde{k}_{\max }^{2}=(1 / 2)\left(\sqrt{(1+\chi)^{2}+4 \chi \eta_{1}}-(1+\chi)\right)(1-\gamma)\right) .
$$

Таким образом, значение волнового вектора, соответствующего максимальному инкременту колебаний уменьшается уже в линейном приближения по диссипативному параметру $\gamma$. При нахождении максимального значения инкремента колебаний, мы будем полагать в (35) $\gamma=0$, поскольку учет $\gamma$ в этом выражении приводит к квадратичным поправкам по этому параметру в инкременте колебаний, что выходит за рамки принятого приближения. Максимальный инкремент будем находить из выражения (31) для $\tilde{G}_{1 \max }$ при волновом векторе $\tilde{k}^{2}=\left(\tilde{k}_{1}\right)_{\max }^{2}$

$$
\tilde{G}_{1 \max }=\hbar G_{1 \max } /\left(n_{10} g_{1}\right)=\sqrt{\left(\tilde{k}_{1}\right)_{\max }^{2}\left(\sqrt{(1+\chi)^{2}+4 \chi \eta_{1}}-\left(\tilde{k}_{1}\right)_{\max }^{2}-(1+\chi)\right)}=\left(\tilde{k}_{1}\right)_{\max }^{2} .
$$

И, соответственно, инкремент $G_{1 \max }$ равен

$$
G_{1 \max }=\hbar\left(k_{1}\right)_{\max }^{2} / 2 m
$$

Здесь, согласно (35) (при $\gamma=0$ )

$$
\left(k_{1}\right)_{\max }^{2}=\left(m / \hbar^{2}\right)\left(\sqrt{\left(n_{10} g_{1}+n_{20} g_{2}\right)^{2}+4 n_{10} n_{20} g_{1} g_{2} \eta_{1}}-\left(n_{10} g_{1}+n_{20} g_{2}\right)\right) .
$$

Найденное максимальное значение инкремента (37) это известный результат, полученный при описании модуляционной неустойчивости в БЭК с положительными константами связи в каждой компоненте [17].

Исследуем, в каких пределах волновых векторов существует МН, в случае, когда $g_{1}>0, g_{2}<0$, т.е. когда постоянная взаимодействия во второй компоненте отрицательна. Перепишем выражение (25) в виде

$$
\left(E_{0}^{ \pm}\right)^{2}=\tilde{k}^{2}\left(\tilde{k}^{2}+(1-|\chi|) \pm \sqrt{(1-|\chi|)^{2}+4|\chi| \eta_{2}}\right),
$$

где $|\chi|=n_{2}\left|g_{2}\right| / n_{1} g_{1}, \eta_{2} \equiv 1+g_{12}^{2} /\left(g_{1}\left|g_{2}\right|\right)$.

Здесь параметр $\eta_{2}>0$. Из (39) видно, что $\left(E_{0}^{+}\right)^{2}>0$ для любых значений параметра $\chi$. Таким образом, МН в этом случае отсутствует. Для второй ветви колебаний с частотами $\omega_{0}^{-}$возможно как $\left(E_{0}^{-}\right)^{2}>0$, так и $\left(E_{0}^{-}\right)^{2}<0$. Для значений волновых векторов $\tilde{k}^{2}<\tilde{k}_{B 2}^{2}$

$$
\tilde{k}_{B 2}^{2}=|\chi|-1+\sqrt{(|\chi|-1)^{2}+4|\chi| \eta_{2}}
$$

выполняется неравенство $\left(E_{0}^{-}\right)^{2}<0$ и в системе, в отсутствие диссипации, будет существовать неустойчивость с инкрементом колебаний $G_{02}$, следующим из выражения для $\tilde{G}_{02}\left(\tilde{G}_{02} \equiv \hbar G_{02} /\left(n_{10} g_{1}\right)\right)$

$$
\tilde{G}_{02}=\sqrt{\tilde{k}^{2}\left(\sqrt{(|\chi|-1)^{2}+4|\chi| \eta_{2}}+|\chi|-1-\tilde{k}^{2}\right)}
$$

Заметим, что в отличие от случая, когда внутри компонент конденсата постоянные взаимодействия между частицами положительны и для развития МН необходимо выполнение условия $g_{12}^{2}-g_{1} g_{2}>0$, здесь, для возникновения $\mathrm{MH}$, на соотношение между величинами $g_{1},\left|g_{2}\right|, g_{12}$ не накладывается никаких условий (см.[12]). При наличии диссипации граничное значение волнового вектора для существования МН определяется уравнениями (24) и (39) и, в линейном по $\gamma$ приближении, остается прежним (40). В размерных единицах

$$
k_{B 2}^{2}=\left(2 m / \hbar^{2}\right)\left[\sqrt{\left(n_{10} g_{1}-n_{20}\left|g_{2}\right|\right)^{2}+4 n_{10} n_{20}\left(g_{12}^{2}+g_{1}\left|g_{2}\right|\right)}-\left(n_{10} g_{1}-n_{20}\left|g_{2}\right|\right)\right] \text {. }
$$


Аналогично первому случаю с константами взаимодействия $g_{1}>0, g_{2}>0$, выражение для инкремента колебаний в присутствии диссипации при $g_{1}>0, g_{2}<0$ и волновых векторах $0<\tilde{k}^{2}<\tilde{k}_{B 2}^{2}$ определяется формулой (28), но значения $E_{0}^{-}$находятся из (39). В результате получаем

$$
\tilde{G}_{2}=\tilde{G}_{02}-\frac{\gamma}{2}\left(\tilde{k}^{2}+\frac{\left(E_{0}^{-}\right)^{2}}{\tilde{k}^{2}}\right)=0 .
$$

Таким образом, влияние диссипации на $\mathrm{MH}$ в этом случае будет определяться слагаемым $(\gamma / 2)\left(2 \tilde{k}^{2}+(1-|\chi|)-\sqrt{(1-|\chi|)^{2}+4|\chi| \eta_{2}}\right)$ с параметрами $|\chi|$ и $\eta_{2}$. Приведем выражение для инкремента колебаний $G_{2}$, возвращаясь к размерным величинам

$$
G_{2}=G_{02}-\frac{\gamma}{2 \hbar}\left(2 \varepsilon_{0}+\left(n_{10} g_{1}-n_{2}\left|g_{2}\right|\right)-\sqrt{\left(n_{10} g_{1}-n_{2}\left|g_{2}\right|\right)^{2}+4 n_{10} n_{20} g_{1}\left|g_{2}\right| \eta_{2}}\right),
$$

где инкремент $G_{02}$ согласно (41)

$$
G_{02}=(1 / \hbar) \sqrt{\varepsilon_{0}\left(\sqrt{\left(n_{10} g_{1}-n_{2}\left|g_{2}\right|\right)^{2}+4 n_{10} n_{20} g_{1}\left|g_{2}\right| \eta_{2}}+\left(n_{20}\left|g_{2}\right|-n_{10} g_{1}\right)-\varepsilon_{0}\right)} .
$$

Моды колебаний, при которых достигается максимальное значение инкремента, при различных значениях параметров $|\chi|$ и $\eta_{2}$, определяются условием (34). Соответствующие волновые вектора и инкременты определяются выражениями

$$
\begin{gathered}
\left(\tilde{k}_{2}\right)_{\max }^{2}=(1 / 2)\left(|\chi|-1+\sqrt{(|\chi|-1)^{2}+4|\chi| \eta_{2}}\right) . \\
E_{0}^{-}=\sqrt{\left(\tilde{k}_{2}\right)_{\max }^{2}\left(\left(\tilde{k}_{2}\right)_{\max }^{2}+(1-|\chi|)-\sqrt{(1-|\chi|)^{2}+4|\chi| \eta_{2}}\right)} .
\end{gathered}
$$

Аналогично случаю с положительными константами связи получим для случая $g_{1}>0, g_{2}<0$

$$
\tilde{G}_{2 \max }=\sqrt{\left(\tilde{k}_{2}\right)_{\max }^{2}\left(\sqrt{(1-|\chi|)^{2}+4|\chi| \eta_{2}}-\left(\tilde{k}_{2}\right)_{\max }^{2}-(1-|\chi|)\right)}=\left(\tilde{k}_{2}\right)_{\max }^{2} .
$$

Или, переходя к размерным единицам

$$
\begin{gathered}
G_{2 \max }=\hbar\left(k_{2}\right)_{\max }^{2} / 2 m, \\
\left(k_{2}\right)_{\max }^{2}=\left(m / \hbar^{2}\right)\left(\sqrt{\left(n_{10} g_{1}-n_{20}\left|g_{2}\right|\right)^{2}+4 n_{10} n_{20} g_{1}\left|g_{2}\right| \eta_{2}}-\left(n_{10} g_{1}-n_{20}\left|g_{2}\right|\right)\right) .
\end{gathered}
$$

Заметим, что при нахождении максимальных инкрементов $G_{1 \max }$ и $G_{2 \max }$, в рассматриваемых нами случаях

$$
\frac{\partial\left(E_{0}^{-}\right)^{2}}{\partial \tilde{k}^{2}}=2\left(\tilde{k}^{2}-\tilde{k}_{\max }^{2}\right)=\tilde{k}^{2}+\frac{\left(E_{0}^{-}\right)^{2}}{\tilde{k}^{2}} .
$$

Отсюда следует, что декремент колебаний $\tilde{\Gamma}^{-}=(\gamma / 2 \hbar)\left(\tilde{k}^{2}+\left(E_{0}^{-}\right)^{2} / \tilde{k}^{2}\right)$, обусловленный диссипацией, в точности равен нулю в точке максимального инкремента модуляционной неустойчивости. Таким образом, диссипация, имеющаяся в системе и приводящая к затуханию возбуждений устойчивых ветвей колебаний, в случае развития МН, не приводит даже к частичному подавлению самой быстро растущей моды колебаний, оставляя значение частоты $\omega_{0}^{-}$для волновых векторов $k_{\max }^{2}$ неизменным

$$
\begin{gathered}
E^{-}=\left[\left(E_{0}^{-}\right)-i \gamma\left(\tilde{k}^{2}-\tilde{k}_{\max }^{2}\right)\right]_{\tilde{k}^{2}=\tilde{k}_{\max }^{2}}=E_{0}^{-} . \\
\left(\omega^{-}\right)_{k^{2}=k_{\max }^{2}}=\omega_{0}^{-} .
\end{gathered}
$$

Рассмотрим длинноволновое приближение, когда использование уравнения ГП наиболее обоснованно. Поскольку $\lambda>>, \xi=\hbar / \sqrt{2 m n g}$ - длина когерентности в БЭК, то $\tilde{k}^{2}<<1$ в данном приближении. Перейдем к размерным переменным и выпишем, согласно (22), (23) спектр колебаний в длинноволновой области

$$
\begin{aligned}
& \omega^{ \pm}=k \sqrt{(1 / 2 m)\left(n_{1} g_{1}+n_{2} g_{2} \pm \sqrt{\left(n_{1} g_{1}+n_{2} g_{2}\right)^{2}+4 n_{1} n_{2}\left(g_{12}^{2}-g_{1} g_{2}\right)}\right.}- \\
& -\frac{i \gamma}{2 \hbar}\left(n_{1} g_{1}+n_{2} g_{2} \pm \sqrt{\left(n_{1} g_{1}+n_{2} g_{2}\right)^{2}+4 n_{1} n_{2}\left(g_{12}^{2}-g_{1} g_{2}\right)}\right)
\end{aligned} .
$$

Или, вводя скорости звука в компонентах, $C_{1}=\sqrt{g_{1} n_{10} / m}$ и $C_{2}=\sqrt{g_{2} n_{20} / m}$ 


$$
\omega^{ \pm}=k \sqrt{\frac{C_{1}^{2}+C_{2}^{2}}{2} \pm \sqrt{\left(\frac{C_{1}^{2}+C_{2}^{2}}{2}\right)^{2}+C_{1}^{2} C_{1}^{2}\left(g_{12}^{2} /\left(g_{1} g_{2}\right)-1\right)}}-\frac{i \gamma m}{\hbar}\left[\frac{C_{1}^{2}+C_{2}^{2}}{2} \pm \sqrt{\left(\frac{C_{1}^{2}+C_{2}^{2}}{2}\right)^{2}+C_{1}^{2} C_{1}^{2}\left(g_{12}^{2} /\left(g_{1} g_{2}\right)-1\right)}\right]
$$

Отсюда следует, что зависимость частоты колебаний от волнового вектора имеет качественно такой же вид, как и для однокомпонентного конденсата при $\lambda>>\xi, \xi=\hbar / \sqrt{2 m n g}, C=\sqrt{g n / m}$

$$
\omega=k C-i \gamma m C^{2} / \hbar \text {. }
$$

\section{ВЫВОДЫ}

В однокомпонентном БЭК модуляционная неустойчивость существует только тогда, когда постоянная взаимодействия между частицами отрицательна. Внутрикомпонентное и межкомпонентное взаимодействие частиц в двухкомпонентном бозе-конденсате приводит к нетривиальному условию на параметры системы для возникновения МН. Возникновение МН в двухкомпонентном БЭК зависит уже как от знака, так и от величины постоянных взаимодействия между частицами. В случае, когда в компонентах конденсата постоянные межчастичного взаимодействия положительны, возникновение МН определяется двумя безразмерными параметрами: $\chi \equiv\left(n_{2} g_{2}\right) /\left(n_{1} g_{1}\right)$ и $\eta_{1} \equiv g_{12}^{2} /\left(g_{1} g_{2}\right)-1$. Причем, соотношение между величинами $g_{1}, g_{2}, g_{12}$ таково, что знак $\eta_{1}$ должен быть положительным. Когда в первой компоненте константа связи между частицами положительна, а во второй - отрицательна, ситуация меняется. В этом случае для возникновения МН на соотношение между величинами $g_{1},\left|g_{2}\right|, g_{12}$ не накладывается никаких условий.

В работе, в линейном приближении по диссипативному параметру $\gamma$, найдены инкремент колебаний и значение волнового вектора, соответствующего максимальному инкременту колебаний. При этом показано, что границы области волновых векторов, при которых существует $\mathrm{MH}$, остаются такими же как и в бездиссипативном случае. Показано, что для значения волнового вектора, соответствующего максимуму инкремента колебаний, вклад диссипации в линейном приближении равен нулю и необходимо учитывать следующее, квадратичное приближение по коэффициенту диссипации для учета этого вклада.

При отсутствии МН, для устойчивых колебаний определены декременты затухания. Показано, что в этом случае в длинноволновом пределе частота колебаний линейно зависит от волнового вектора, как и в случае однокомпонентного конденсата.

Anatoly Ivashin $@$ https://orcid.org/0000-0001-6026-0208

\section{ORCID IDs}

\section{СПИСОК ЛИТЕРАТУРЫ}

[1]. S. Burger, K. Bongs, S. Dettmer, W. Ertmer, K. Sengstock, A. Sanpera, V. Shlyapnikov and M. Levenstein, Phys. Rev. Lett. 53, 5198-5201 (1999).

[2]. B. Eiermann, Th. Anker, M. Albiez, M. Taglieber, P. Treutlein, K.-P.Marzlin and K. Oberthaler, Phys. Rev. Lett. 92, 230401 (2004).

[3]. M.R. Matthews, B.P. Anderson, P.S. Haljan, D.S. Hall, C.E. Wieman and E.A. Cornell, Phys. Rev. Lett. 83, $2498-2501$ (1999).

[4]. G.P. Agrawal, Phys. Rev. Lett. 59, 880-883 (1987).

[5]. E.A. Donley, N.R. Claussen, S.L. Cornish, J.L. Roberts and E.A. Cornell, Nature, 412, 295-299 (2001).

[6]. K.E. Strecker, G.B. Partridge, A.G. Truscott and R.G. Hulet, Nature, 417, 150-153 (2002).

[7]. H.-J. Miesner, D.M. Stamper-Kurn, J. Stenger, S. Inouye, A.P. Chikkatur and W. Ketterle, Phys. Rev. Lett. 82, 2228-2231 (1999).

[8]. T.L. Ho and V.B. Shenoy, Phys. Rev. Lett. 77, 3276-3279 (1996).

[9]. H. Saito and M. Ueda, Phys. Rev. Lett. 86, 1406-1409 (2001).

[10]. E.W. Goldstein and P. Meystre, Phys. Rev. A. 55, 2935 - 2940 (1997).

[11]. L.D. Carr and J. Brand, Phys. Rev. Lett. 92, 040401 (2004).

[12]. K. Kasamatsu and M. Tsubota, Phys. Rev. A. 74, 013617 (2006).

[13]. S. Ronen, J.L. Bohn, L.E. Halmo and M. Edwards, Phys. Rev. A. 78, 053613 (2008).

[14]. Coen S. and M. Haelterman, Phys. Rev. Lett. 87(1-4), 140401 (2001).

[15]. Yu.M. Poluektov, Low Temp. Phys. 40, 389-398 (2014).

[16]. C.J. Pethick and H. Smith, Bose-Einstein Condensation in Dilute Gases, 2nd ed. (Cambridge University Press, Cambridge, 2008$)$, p. 569.

[17]. P. Ao and S.T. Chui, Phys. Rev. A. 58, 4836-4840 (1998), arXiv: 0003185 [cond-mat]. 